\title{
Nächtliche Langzeitregistrierung von Lungengeräuschen bei Patienten mit gastroösophagealem Reflux
}

\author{
Nocturnal Long-Term Monitoring of Lung Sounds in Patients with Gastro-Oesophageal Reflux Disease
}

Autoren

Institute
P. Lenniger ${ }^{1}$, V. Gross ${ }^{1}$, S. Kunsch ${ }^{2}$, C. Nell ${ }^{2}$, J. E. S. Nolte ${ }^{1}$, A. K. Sohrabi ${ }^{1}$, U. Koehler ${ }^{1}$

Klinik für Innere Medizin, SP Pneumologie, Intensiv- und Schlafmedizin (Direktor: Prof. Dr. C. Vogelmeier)

2 SP Gastroenterologie und Endokrinologie (Direktor: Prof. Dr. T. Gress), Philipps-Universität Marburg eingereicht 10.10 .2009

akzeptiert 20.10.2009

\section{Bibliografie}

Dol http://dx.doi.org/ 10.1055/s-0029-1215344

Online-Publikation: 2. 12.2009

Pneumologie 2010; 64:

255-258 @ Georg Thieme

Verlag KG Stuttgart · New York

ISSN 0934-8387

\section{Korrespondenzadresse}

Prof. Dr. U. Koehler

Universitätsklinikum Gießen

und Marburg $\mathrm{GmbH}$

Standort Marburg

Baldingerstraße 1

35033 Marburg

koehleru@med.uni-marburg.de

\section{Zusammenfassung \\ $\nabla$}

Die gastroösophageale Refluxerkrankung (GERD) ist eine der häufigsten Erkrankungen in den Wohlstandsländern Europas und Nordamerikas. Vor allem die pulmonalen Manifestationen dieser Erkrankung sind in den letzten Jahren in den Mittelpunkt des klinischen und wissenschaftlichen Interesses gerückt. Obgleich die hohe Koinzidenz zwischen Refluxerkrankung und pulmonalen Erkrankungen wie Asthma bronchiale und dem chronischen Husten unbestritten ist, konnte der kausale Zusammenhang nicht eindeutig belegt werden. Wir stellen in dieser Arbeit das Marburger Lung-Sound-Monitoring als neue Methode zur Detektion der nächtlichen respiratorischen Symptome Husten, Wheezing und Räuspern in zeitlich exakter Korrelation mit Refluxereignissen vor. Diese Methode wird es uns zukünftig erlauben, das zeitliche Zusammenspiel von Refluxereignissen, deren Ausprägung bezüglich Zeitdauer und Intensität sowie respiratorischen Symptomen methodisch exakt zu beschreiben.

\section{Gastroösophageale Refluxkrankheit (GERD)}

Die gastroösophageale Refluxkrankheit (GERD) zählt zu den häufigsten Krankheiten der westlichen Welt. Die Prävalenz beträgt ca. 10-20\% der Bevölkerung [1,2]. Definitionsgemäß liegt eine GERD dann vor, wenn durch Reflux von Magenoder Dünndarminhalt klinische Beschwerden hervorgerufen und/oder organische Veränderungen der Speiseröhre induziert werden $[3,4]$. Die Pathophysiologie ist multifaktoriell. Prädisponierende Faktoren sind die Insuffizienz des unteren Ösophagussphinkters, transiente Sphinkterrelaxationen oder auch das Vorliegen von axialen Hiatushernien. Zudem besteht die Möglichkeit, dass es im Rahmen einer bronchialen Obstruktion

\section{Abstract \\ $\nabla$}

Gastro-oesophageal reflux disease (GERD) is one of the most common clinical conditions in the developed countries. Particular interest in pulmonary manifestations of this disease has arisen over the last few years. Although the high coincidence between reflux and chronic cough is unquestioned, the proof of a causal correlation is still lacking. In this paper we present the Marburger Lung-Sound-Monitoring as a new method for the detection of nocturnal respiratory symptoms such as cough, wheezing and throat clearing and their temporal correlation with reflux. This method will in future allow us to precisely record and to evaluate the extent and duration of reflux events and their correlation with respiratory symptoms. vermehrt zu Refluxereignissen kommen kann. Dies wird mit einer Herabsetzung des transdiaphragmalen Drucks erklärt [5].

Als Goldstandard in der Diagnostik des gastroösophagealen Refluxes hat sich die 24-Stunden-phMetrie bewährt [6,7]. Eine pH-Messsonde wird transnasal im Ösophagus platziert, die dann mithilfe eines Rekorders den pH-Wert über $24 \mathrm{~h}$ aufzeichnet. Bei Vorliegen respiratorischer Symptome werden bevorzugt Sonden mit zwei Messpunkten verwendet. Die proximale ist pharynxnah gelegen und dient zum Nachweis eines hohen Refluxes mit der Gefahr einer Aspiration [8]. Symptome wie Husten, Luftnot oder saures Aufstoßen können als Ereignisse dokumentiert und anschließend mit den Befunden der pH-Metrie verglichen werden. 


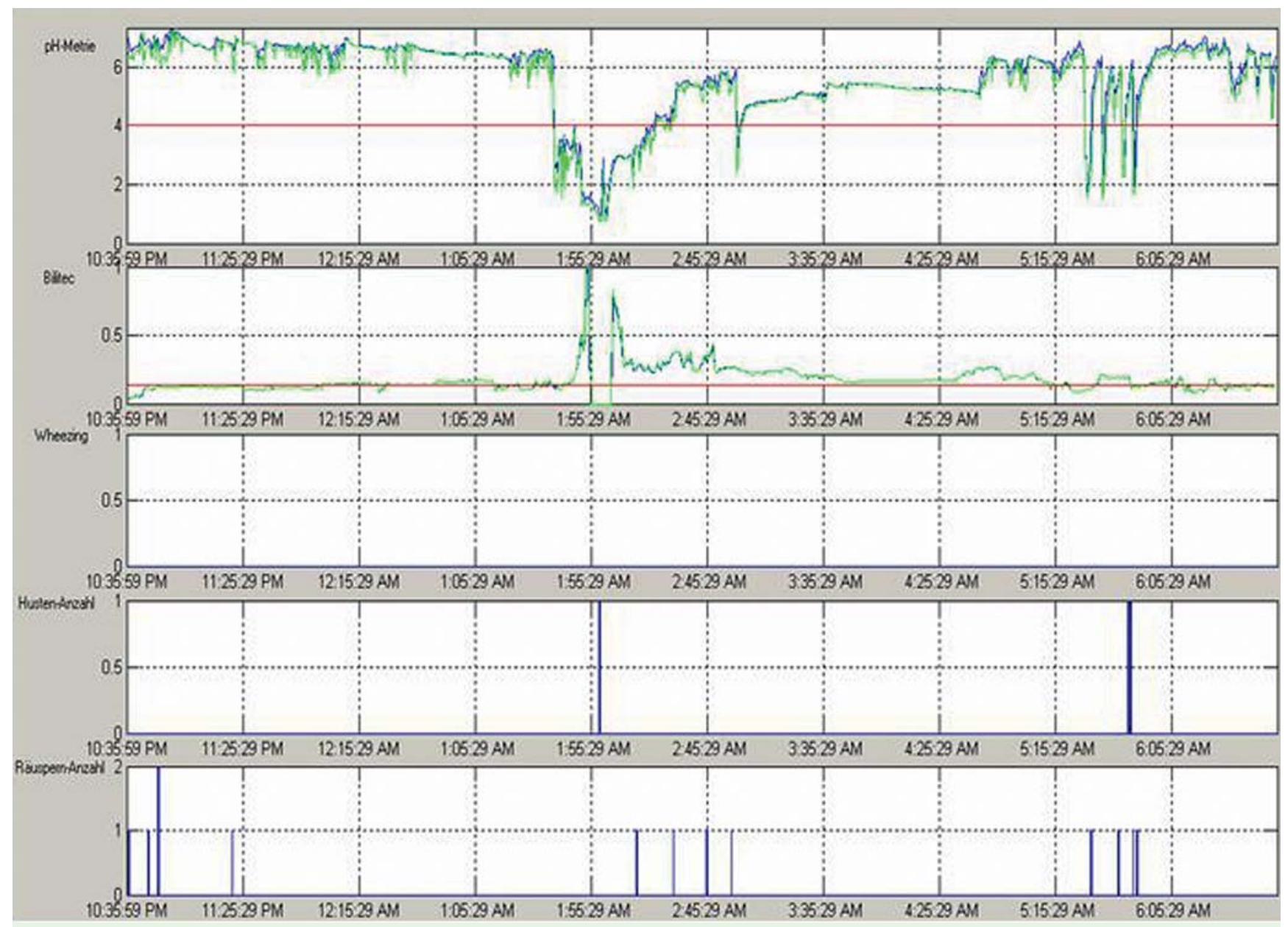

Abb.1 Gegen 1.30 Uhr imponiert bei dem 55-jährigen männlichen Patienten eine lange Refluxepisode mit sauren und biliär dominierten Anteilen, einhergehend mit einem Hustenereignis und dem Auftreten von vermehrtem Räuspern. In der Zeit zwischen 5.25 und 5.50 Uhr zeigen sich vier repetitive Refluxphasen, die ebenfalls von einem Hustenereignis und Räuspern begleitet werden (dargestellt von oben nach unten sind: ph-Metrie; Bilitec-Messung, Wheezing (Giemen); Husten und Räuspern.

Neben dem sauren Reflux wurde in den letzten Jahren zunehmend die Bedeutung des duodeno-gastro-ösophagealen Refluxes (DGER) als Risikofaktor für den Verlauf und die Prognose der GERD erkannt, wobei Gallensäuren als wichtigste Komponente angesehen werden. Mit der Einführung des Bilitec 2000 ist es mittlerweile möglich, den DGER indirekt photometrisch qualitativ und quantitativ über 24 Stunden zu messen. Die ösophageale Impedanzmessung mit pH-Metrie stellt eine weitere vielversprechende Innovation in der Refluxdiagnostik dar. Diese erlaubt nichtsaure und gasförmige Refluxereignisse und deren Bewegungsrichtung zu erfassen [9].

\section{Respiratorische Symptome bei GERD}

Die gastroösophageale Refluxkrankheit bietet eine Vielzahl verschiedener Symptome. Häufig sind die typischen ösophagealen Symptome wie Sodbrennen, saures Aufstoßen, Dysphagie und Regurgitationen [10]. Aber auch extraösophageale Symptome können auf das Vorliegen einer GERD hinweisen [11,12]. Vor allem die pulmonalen Manifestationen dieser Erkrankung wie chronischer Husten oder das Wheezing als Ausdruck der bronchialen Konstriktion werden häufig fehlinterpretiert und stellen eine besondere diagnostische Herausforderung dar. Von HNO- ärztlicher Seite wird der gastroösophageale Reflux mit der posterioren Laryngitis, der sekretorischen Pharyngitis, Stimmbandgranulomen sowie der chronischen Sinusitis pathogenetisch in Zusammenhang gebracht. Auch Zahnerosionen, Zungenbrennen sowie eine Lungenfibrose müssen an das Vorliegen einer GERD denken lassen [5].

Eine GERD lässt sich bei 30 bis $80 \%$ der Asthmatiker nachweisen. Neben Asthma bronchiale und Nasennebenhöhlenerkrankungen gilt die GERD als dritthäufigste Ursache eines chronischen Hustens, mit einem geschätzten Anteil von 20\% [12]. Bei einer Studie von Roka et al. wurden bei 299 Patienten mit einer GERD bei $56(18 \%)$ respiratorische Symptome nachgewiesen. Husten war bei 42/56 (75\%) Patienten auffällig, thorakales Brennen und Regurgitationen bei 30/56 (54\%) bzw. 24/56 (43\%) Patienten. $18 \%$ der Patienten hatten ein Asthma bronchiale [13]. Leggett et al. konnten bei 39 von 52 Patienten (75\%) mit schwer therapierbarem Asthma bronchiale eine GERD nachweisen [14]. Aufgrund der hohen Koinzidenz zwischen GERD und respiratorischen Symptomen scheint ein Zusammenhang naheliegend. Eine Kausalität konnte jedoch bislang methodisch bedingt nicht nachgewiesen werden. 


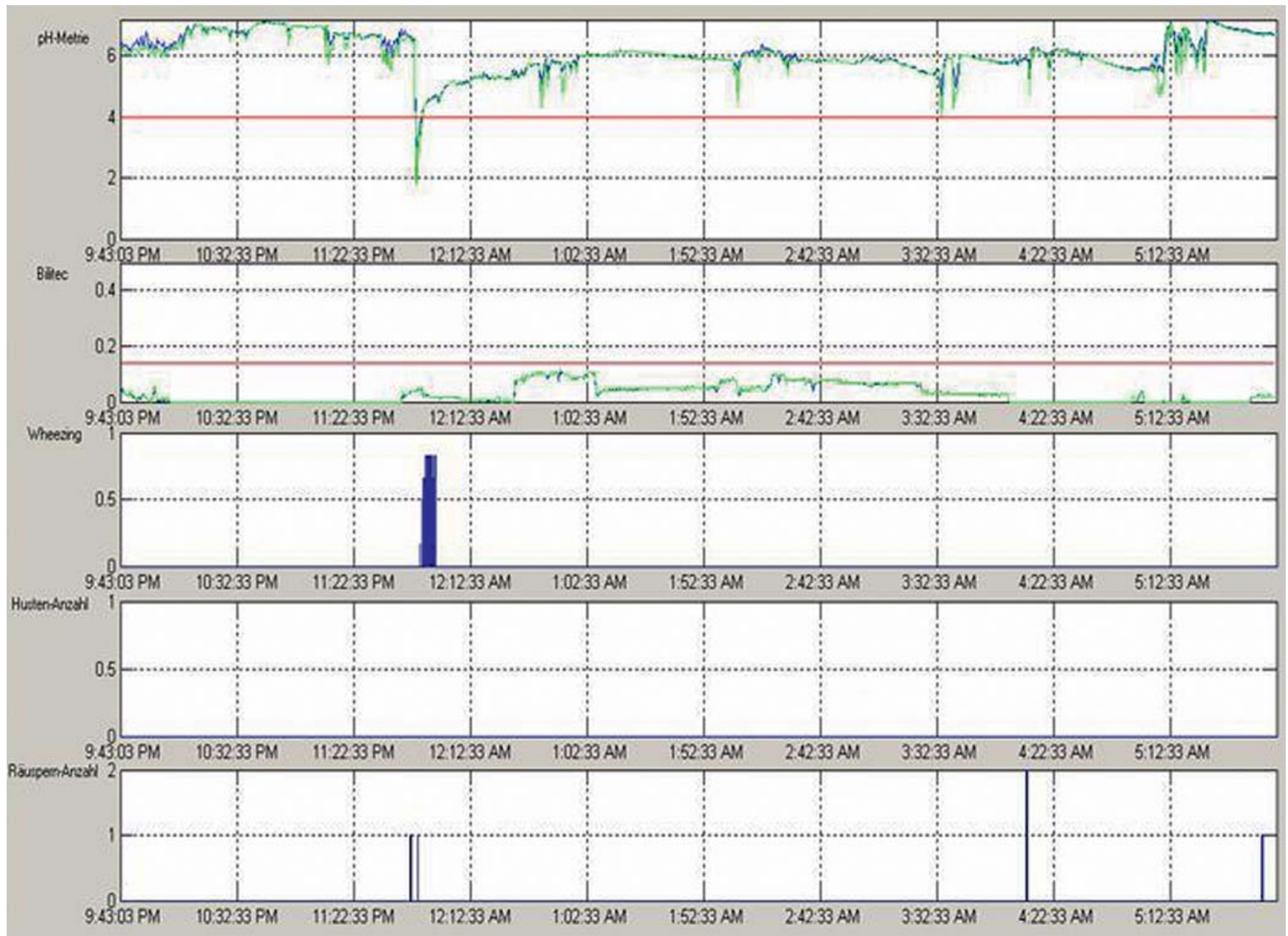

Abb. 2 Messung bei einem 50-jährigen Patienten: Gegen 23.50 Uhr zeigt sich das Auftreten eines isolierten sauren Refluxes, dem zeitnah Wheezing über mehr als fünf Minuten folgt. Hustenereignisse sind nicht zu objektivieren, lediglich vereinzeltes Räuspern.

\section{Wechselwirkung GERD, Atmung und Schlaf}

$\nabla$

Als ursächlich für die unter ösophagealer Säureexposition auftretenden respiratorischen Symptome wie Husten, Wheezing (Giemen) und Dyspnoe werden neben vagal vermittelten ösophagotracheobronchialen Reflexmechanismen Mikroaspirationen ins Bronchialsystem diskutiert $[2,7,15]$. Diese Hypothese wird durch experimentelle Untersuchungen gestützt, bei denen nachgewiesen werden konnte, dass es durch intraösophageale Säureinstillation zu Bronchialobstruktionen kommt $[16,17]$.

Es ist bekannt, dass im Schlaf auftretende Refluxereignisse die Schlafqualität nachhaltig stören können. Nach einer Analyse der American Gastroenterological Association haben 79\% der Patienten, die mindestens einmal pro Woche unter Sodbrennen leiden, auch Symptome in der Nacht. Von den Patienten mit nächtlichem Sodbrennen klagen 75\% über Schlafstörungen, die sich als Einund Durchschlafstörungen manifestieren [18]. Es ist zu mutmaßen, dass der Reflux von Magen- bzw. Dünndarmsekret eine Arousalreaktion triggert, die konsekutiv die Clearancemechanismen aktiviert und gleichzeitig die Schlafstruktur stört. Im Schlaf verändert sich die Ösophagusmotilität in Abhängigkeit der Schlafstadien. Ösophaguskontraktionen treten häufiger im REMund Leichtschlaf auf, im Tiefschlaf sind sie seltener. Die SäureClearancezeit des Ösophagus im Schlaf ist durch eine geringere Schluckfrequenz bei zudem verminderter Speichelproduktion verlängert. Voraussetzung für das Auftreten eines Refluxes ist die Kombination aus insuffizienter Antirefluxbarriere, gestörter Clearance des Ösophagus sowie einem aggressiven Refluat. Intraabdominelle Drucksteigerungen, beispielsweise bei Adipositas permagna, obstruktiver Schlafapnoe, Körperlagewechsel oder Hustenereignisse können eine Insuffizienz der Antirefluxbarriere verstärken.

\section{Die nächtliche Langzeitregistrierung von Lungengeräuschen}

Obgleich an der hohen Koinzidenz von GERD und respiratorischen Symptomen keine Zweifel bestehen, konnte der exakte zeitliche Zusammenhang zwischen Refluxereignissen und nächtlichen respiratorischen Symptomen bisher nicht untersucht werden. Während sich die bisherigen Bemühungen auf die Identifikation respiratorischer Symptome durch Patiententagebücher, subjektive quantitative Patientenaussagen oder Ereignisdokumentation während einer kontinuierlichen ösophagealen $\mathrm{pH}$ Messung bezogen, ist es mit der von uns neu entwickelten Methode der Langzeitregistrierung der Lungengeräusche nun erstmals möglich geworden, nächtliche respiratorische Symptome im Langzeitverlauf und zeitlich synchron zur pH-Metrie und Bilitec-Messung zu erfassen. Gerade die Schlafphase mit der Problematik des „nächtlichen Asthma bronchiale“ sowie der Verschlechterung der Atmung bei Patienten mit chronischer Sinusi- 
tis und postnasal drip-Syndrom kann im Hinblick auf das Vorliegen von respiratorischen Symptomen problemlos dargestellt werden. Eine Kombination aus polysomnografischer Diagnostik, Lungengeräusch- und Refluxaufzeichnung erlaubt zudem die Beurteilung der Einflussnahme von Refluxereignissen auf die Makro- und Mikrostruktur des Schlafes.

Basierend auf dem CORSA-Standard wurde von unserer Arbeitsgruppe ein mobiles System zur Langzeitaufzeichnung von Lungengeräuschen entwickelt [19]. Das derzeit eingesetzte System besteht aus 4 Flachmikrofonen. Zwei werden über den basalen Anteilen der Lunge fixiert, ein weiteres über der Trachea. Ein viertes Mikrofon dient der Aufzeichnung von Außengeräuschen. Die Aufzeichnung der Geräusche erfolgt mit einem kleinen Datenlogger mit integrierter Filter- und Verstärker-Elektronik. Mittels eines Analog-zu-Digital-Wandlers werden die Geräusche zur Speicherung auf einem Computer umgewandelt. Ein eigens entwickeltes Programm, basierend auf Techniken der Biosignal-Analyse, dient zur grafischen Wiedergabe und audiovisuellen Auswertung der aufgezeichneten Geräusche [20].

\section{Akustische Langzeitregistrierung, pH-Metrie und Bilitec-Messung im Schlaf}

Im Folgenden soll anhand von zwei Fallbeispielen ( $\bullet$ Abb. 1 und 2) die kombinierte Messung von ph-Metrie, Bilitec und Lungengeräuschen demonstriert werden. Die Diagramme beinhalten die Daten der 24h-pH-Metrie, der Bilitec-Messung und der Lungengeräuschaufzeichnung im zeitlichen Verlauf. Die akustisch aufgezeichneten Phänomene sind Wheezing (Giemen), Husten und Räuspern. Die pathologischen Grenzen der ph-Metrie liegen bei einem pH-Wert kleiner 4, die der Bilitec-Messung bei einer Bilirubinabsorption größer 0,14. Wheezing-Ereignisse werden ab einer Dauer von 2 min als klinisch relevant definiert.

\section{Zusammenfassung - Was ist das Besondere der neuen Methode? \\ $\nabla$}

Die audiovisuelle Darstellung der nächtlichen Lungengeräusche mittels Lung-Sound-Monitoring in Kombination mit einer $24 \mathrm{~h}-$ ph-Metrie mit Bilitec-Untersuchung ermöglicht erstmals die Darstellung eines zeitlichen Zusammenhangs zwischen Refluxereignissen, deren Ausprägung bezüglich Zeitdauer und Intensität, und pulmonaler Manifestationen wie Husten, Wheezing und Räuspern.

Obgleich großangelegte Studien mit selektionierten Patienten noch ausstehen, zeigen erste Patientenmessungen vielversprechende Ergebnisse. Somit wird es zukünftig möglich sein, das pathophysiologische Zusammenspiel zwischen Reflux und respiratorischen Symptomen exakt zu beschreiben. Mit Erweiterung der Methodik um die ösophageale Impedanzmessung können auch der nicht saure und gasförmige Reflux in die Untersuchungen mit einbezogen werden. Ziel ist die Identifikation von Patienten mit einem kausalen Zusammenhang von Reflux und respiratorischen Symptomen.

\section{Interessenkonflikte}

Die Autoren geben an, dass kein Interessenkonflikt besteht.

\section{Literatur}

1 Asano K, Suzuki H. Silent acid reflux and asthma control. N Engl J Med 2009; 360: 1551 - 1553

2 Mansfield LE, Hameister HH, Spaulding HS et al. The role of the vagus nerve in airway narrowing caused by intraesophageal hydrochloric acid provocation and esophageal distension. Ann Allergy 1981; 47: $431-434$

3 Kunsch S, Linhart T, Fensterer $H$ et al. Prävalenz eines pathologischen Duodeno-Gastro-Ösophagealen Refluxes (DGER) bei Patienten mit klinischen Beschwerden einer Refluxerkrankung. Z Gastroenterol 2008; 46: $409-414$

4 Nepomnyashchikh DL, Aidagulova SV, Korabel'nikov DI et al. Ultrastructural changes in the epithelium in asthma associated with gastroesophageal reflux. Bull Exper Biol Med 2007; 144: 253 - 257

5 Hellmig S, Fölsch UR. Gastroösophageale Refluxerkrankung: Diagnostik und Therapie einer Volkskrankheit. Med Klin 2007; 120: 373 - 380

6 Shay SS, Johnson LF, Richter JE. Acid rereflux: A review, emphasizing detection by impedance, manometry, and scintigraphy, and the impact on acid clearing pathophysiology as well as interpreting the $\mathrm{pH}$ record. Dig Dis Sci 2003; 48: 1 -9

7 Wenzl TG, Moroder C, Trachterna $M$ et al. Esophageal pH monitoring and impedance measurement: a comparison of two diagnostic tests for gastroesophageal reflux. J Pediatr Gastroenterol Nutr 2002; 34: $519-523$

8 Galmiche JP, Zerbib F, Des Varannes B. Review article: respiratory manifestations of gastro-oesophageal reflux disease. Aliment Pharmacol The 2008; 27: 449-464

9 Huggins S. The role of gastroesophageal reflux disease in asthma. J Am Academy Nurs Practitioners 2008; 20: 238-242

10 Fass R, Quan SF, O'Connor GT et al. Predictors of heartburn during sleep in a large prospective cohort study. Chest 2005; 127: 1658-1666

11 Boesch RP, Daines C, Willging JP et al. Advances in the diagnosis and management of chronic pulmonary aspiration in children. Eur Respir J 2006; 28: $847-861$

12 Poelman J, Tack J. Extraoesophageal manifestations of gastro-eosophageal reflux. Gut 2005; 54: $1492-1499$

13 Róka R, Rosztóczy A, Izbéki F et al. Prevalence of respiratory symptoms and diseases associated with gastroesophageal reflux disease. Digestion 2005; 71: $92-96$

14 Leggett JJ, Johnston BT, Mills $M$ et al. Prevalence of gastroesophageal reflux in difficult asthma: Relationship to asthma outcome. Chest 2005; 127: $1227-1231$

15 Altschuler SM. Laryngeal and respiratory protective reflexes. Am J Med 2001; 111: 90s-94s

16 Araujo ACS, Aprile LRO, Dantas RO et al. Bronchial responsiveness during esophageal acid infusion. Lung 2008; 186: $123-128$

17 King AL, Anggiansah A, Anggiansah R et al. Acid perfusion test: A useful test for evaluating esophageal acid sensitivity. Digestive Diseases and Sciences 2005; 50: 1611 - 1615

18 Shaker R, Castell DO, Schoenfeld PS et al. Nighttime heartburn is an under-appreciated clinical problem that impacts sleep and daytime function: The results of a Gallup survey conducted on behalf of the American Gastroenterological Association. Am J Gastroenterol 2003; 98: $1487-1493$

19 Earis JE. Current methods used for computerized respiratory sound analysis. Eur Respir Rev 2000; 10: 586-590

20 Gross V, Reinke C, Dette F et al. Mobile nocturnal long-term monitoring of wheezing and cough. Biomed Tech 2007; 52: 73-76 